\title{
Effect of severe renal impairment on the pharmacokinetics of brigatinib
}

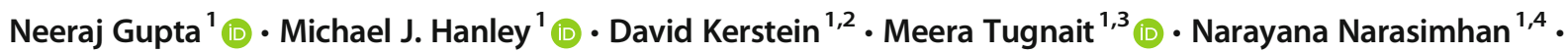 \\ Thomas C. Marbury ${ }^{5}$ (D) Karthik Venkatakrishnan ${ }^{1,6}{ }_{\mathbb{C}}$
}

Received: 1 February 2021 / Accepted: 4 March 2021 / Published online: 20 March 2021

(C) The Author(s) 2021

\section{Summary}

Background Brigatinib, a next-generation anaplastic lymphoma kinase (ALK) inhibitor, targets activated, mutant forms of ALK and overcomes mechanisms of resistance to the ALK inhibitors crizotinib, ceritinib, and alectinib. Brigatinib is approved in multiple countries for treatment of patients with ALK-positive non-small cell lung cancer. Based on population pharmacokinetic (PK) analyses, no dosage adjustment is required for patients with mild or moderate renal impairment. Methods An open-label, single-dose study was conducted to evaluate the PK of brigatinib $(90 \mathrm{mg}$ ) in patients with severe renal impairment (estimated glomerular filtration rate $<30 \mathrm{~mL} / \mathrm{min} / 1.73 \mathrm{~m}^{2} ; \mathrm{n}=8$ ) and matched healthy volunteers with normal renal function (estimated glomerular filtration rate $\geq 90 \mathrm{~mL} / \mathrm{min} / 1.73 \mathrm{~m}^{2} ; \mathrm{n}=8$ ). Plasma and urine were collected for the determination of plasma protein binding and estimation of plasma and urine PK parameters. Results Plasma protein binding of brigatinib was similar between patients with severe renal impairment (92\% bound) and matched healthy volunteers with normal renal function ( $91 \%$ bound). Unbound brigatinib exposure (area under the plasma concentration-time curve from time zero to infinity) was approximately $92 \%$ higher in patients with severe renal impairment compared with healthy volunteers with normal renal function. The renal clearance of brigatinib in patients with severe renal impairment was approximately $20 \%$ of that observed in volunteers with normal renal function. Conclusions These findings support a brigatinib dosage reduction of approximately $50 \%$ in patients with severe renal impairment.

Trial registry: Not applicable.

Keywords Brigatinib $\cdot$ Anaplastic lymphoma kinase $\cdot$ Pharmacokinetics $\cdot$ Renal impairment

Neeraj Gupta

Neeraj.Gupta@takeda.com

$1 \quad$ Millennium Pharmaceuticals, Inc., a wholly owned subsidiary of Takeda Pharmaceutical Company Limited, 40 Landsdowne Street, Cambridge, MA 02139, USA

2 Present address: Anchiano Therapeutics, Cambridge, MA, USA

3 Present address: Spectrum Pharmaceuticals, Inc., Cambridge, MA, USA

4 Present address: Verastem Oncology, Needham, MA, USA

5 Orlando Clinical Research Center, Orlando, FL, USA

6 Present address: EMD Serono, Inc., Billerica, MA, USA

\section{Introduction}

Brigatinib is a next-generation anaplastic lymphoma kinase (ALK) inhibitor that targets a broad range of ALK mutations, including L1196M, F1174C/V, I1171N, and G1202R [1, 2]. Mechanisms of resistance to the ALK inhibitors crizotinib, ceritinib, and alectinib were overcome by brigatinib in nonclinical studies, and brigatinib was found to be a more potent and selective inhibitor of native EML4-ALK compared with these three agents [3]. In the pivotal phase 2 ALTA trial of patients with locally advanced or metastatic ALK-positive non-small cell lung cancer (NSCLC) that progressed on crizotinib, brigatinib $180 \mathrm{mg}$ qd with a seven-day lead-in at $90 \mathrm{mg}$ was associated with an independent review committee-assessed confirmed objective response rate (ORR) of $56 \%$ and median progression-free survival (PFS) of 16.7 months with a median follow-up of 24 months [4]. For patients with baseline brain metastases, brigatinib demonstrated a confirmed intracranial 
ORR of $67 \%$ and median intracranial PFS of 18.4 months. Based on these data, brigatinib was approved for the treatment of patients with metastatic ALK-positive NSCLC with progressive disease on or with intolerance to crizotinib [5]. In a subsequent phase 3 trial (ALTA-1L) in patients with ALK + NSCLC who had not previously received an ALK inhibitor, PFS was significantly longer among patients who received brigatinib than among those who received crizotinib (24-month PFS: blinded independent review committee-assessed, $48 \%$ vs. $26 \%$; hazard ratio, 0.49 [95\% confidence interval [CI], 0.350.68 ]; $P<0.0001$; investigator-assessed, $56 \%$ vs. $24 \% ; 0.43$ [0.31-0.61]; $P<0.0001$ ) [6], leading to expanded approval for first-line use.

The recommended dosing regimen for brigatinib consists of a seven-day lead-in of $90 \mathrm{mg}$ orally once daily (qd) followed by $180 \mathrm{mg}$ qd [5]. This regimen provides a favorable benefit-risk profile by providing a lower starting dose to reduce the frequency of rare early-onset pulmonary events, followed by a higher dose to provide the potential for greater efficacy $[7,8]$. Brigatinib is rapidly absorbed, with a median time to reach maximum plasma concentration $\left(\mathrm{T}_{\max }\right)$ of 1 to $3 \mathrm{~h}$ postdose [7]. Steady-state systemic exposure is dose proportional over the dose range of $60 \mathrm{mg}$ to $240 \mathrm{mg} \mathrm{qd}$ [7], and the mean plasma elimination half-life is $25 \mathrm{~h}$ [5]. Brigatinib has modest drug accumulation with repeat dosing, with a geometric mean accumulation ratio after repeat dosing of 1.9 to $2.4[5,7]$. Brigatinib is primarily metabolized by cytochrome P450 (CYP) 3A4, with minor contribution by 2 C8 in vitro. In the human absorption, distribution, metabolism, and excretion study, $65 \%$ and $25 \%$ of the administered dose was recovered in feces and urine, respectively. Unchanged brigatinib accounted for $41 \%$ and $86 \%$ of the total radioactivity in feces and urine, respectively. Therefore, both hepatic and renal pathways of elimination contribute to the overall clearance of brigatinib in humans [5].

Brigatinib is intended for chronic use in patients with ALK + NSCLC, some of whom may have impaired renal function of varying degrees, especially if they have been previously treated with agents known to be associated with renal toxicity. During development, patients with mild or moderate renal impairment, defined by an estimated glomerular filtration rate (eGFR) of 30 to $<90 \mathrm{~mL} / \mathrm{min} / 1.73$ $\mathrm{m}^{2}$, were allowed to enroll into clinical studies. Based on population pharmacokinetic (PK) analyses, mild or moderate renal impairment were not found to impact brigatinib exposure, thereby indicating that no dose adjustment is required for these patients [9]. Because patients with severe renal impairment $\left(e G F R<30 \mathrm{~mL} / \mathrm{min} / 1.73 \mathrm{~m}^{2}\right)$ were excluded from the pivotal studies, the present study evaluated the PK of brigatinib in patients with severe renal impairment and matched healthy volunteers with normal renal function $\left(\geq 90 \mathrm{~mL} / \mathrm{min} / 1.73 \mathrm{~m}^{2}\right)$ to inform dosing recommendations in this patient population.

\section{Methods}

\section{Study design}

This was an open-label, parallel-group study that enrolled patients with severe renal impairment and matched healthy volunteers with normal renal function. The study consisted of a 28-day screening period and a single nine-day inpatient treatment period (day -1 to day 8). On the morning of day 1 , each participant received a single oral dose of brigatinib $90 \mathrm{mg}$ under fasting conditions.Participants were required to abstain from food for approximately $10 \mathrm{~h}$ prior to study drug administration and for approximately four hours postdose, with the exception of the standardized snack. Due to the high prevalence of diabetes in patients with severe renal impairment, the standardized snack (not high fat or high calorie) was given to all participants at $2.5 \mathrm{~h}$ postdose. Water was permitted ad libitum except for one hour prior to dosing and for approximately one hour postdose. Participants underwent end-ofstudy procedures and were discharged from the clinical site on day 8.

Approval for this study was obtained from the institutional review board of the study site (IntegReview, Austin, Texas, USA) and all participants provided written informed consent. This study was conducted in accordance with current Good Clinical Practice, the Declaration of Helsinki, the International Council for Harmonisation guidelines, and all applicable regulatory requirements.

\section{Participants}

Healthy volunteers with normal renal function (eGFR $\geq 90$ $\mathrm{mL} / \mathrm{min} / 1.73 \mathrm{~m}^{2}$ ) were matched to patients with severe renal impairment by age ( \pm 10 years), sex, body mass index (BMI; $\pm 15 \%$ at screening), and if possible, smoking habits. Patients with severe renal impairment were defined as those with an eGFR $<30 \mathrm{~mL} / \mathrm{min} / 1.73 \mathrm{~m}^{2}$. eGFR was calculated using the Modification of Diet in Renal Disease equation (MDRD) [10]. Estimated creatinine clearance was based on the CockcroftGault equation.

Eligible participants included men or women of nonchildbearing potential aged 18 to 80 years with a BMI of 18.0 to $45.0 \mathrm{~kg} / \mathrm{m}^{2}$ and a minimum weight of $50.0 \mathrm{~kg}$ at screening. Other key inclusion criteria required a nonsmoker or a smoker who was willing to smoke five or fewer cigarettes per day during the inpatient stay. Healthy volunteers with normal renal function were free from any clinically significant abnormality based on medical history, vital signs, physical examination, 12-lead electrocardiogram, and laboratory evaluation at screening.

Patients with severe renal impairment may have had related medical conditions consistent with their disease, such as hypertension and diabetes, that were stable for at least three 
months, but they were excluded if they had a functioning renal transplant or fluctuating or rapidly deteriorating renal function. Use of over-the-counter drugs or herbal supplements, with the exception of occasional acetaminophen $(\leq 2000 \mathrm{mg} /$ day) and vitamins ( $\leq 100 \%$ recommended daily allowance), was not permitted within $72 \mathrm{~h}$ of study drug administration nor was any investigational or prescription drug within 30 days except chronic stable medications taken by patients with renal impairment. Participants were required to abstain from grapefruit and grapefruit-containing products, pomegranate, pomelo, star fruit, poppy seeds, Seville oranges, quinine-containing drinks or foods, and caffeine-containing beverages for $72 \mathrm{~h}$ before study drug administration.

\section{Assessments}

Plasma protein binding Blood samples were collected at 2, 8, and $24 \mathrm{~h}$ after brigatinib administration to determine the plasma protein binding of brigatinib.

Pharmacokinetic measurements Blood samples were collected before brigatinib administration (predose) and at $0.5,1,1.5$, $2,2.5,3,4,6,8,12,24,36,48,60,72,96,120,144$, and $168 \mathrm{~h}$ after brigatinib administration to determine plasma brigatinib concentrations. Urine was collected predose and during intervals $(0-8,8-12,12-24,24-48,48-72,72-96,96-120$, and $120-168 \mathrm{~h}$ ) after brigatinib administration to determine urine brigatinib concentrations. Urine samples were subsequently diluted 50:50 (v:v) urine:isopropanol at the clinical site.

\section{Bioanalytical methods}

\section{Plasma protein binding assay}

Free brigatinib in plasma samples was separated from proteinbound brigatinib by dialyzing the samples across a semipermeable dialysis membrane (8000-Da mass cutoff) using Thermo Scientific ${ }^{\text {TM }}$ Single-Use RED (rapid equilibrium dialysis) Plates. For each RED dialysis unit, an aliquot $(200 \mu \mathrm{L})$ of plasma was transferred into the donor chamber and an aliquot $(350 \mu \mathrm{L})$ of warm $\left(37^{\circ} \mathrm{C}\right)$ phosphate buffered saline (PBS) was transferred into the receiver chamber of the dialysis unit. The RED devices were incubated at $37{ }^{\circ} \mathrm{C}$ with gentle shaking ( $250 \mathrm{rpm}$ on a rotator shaker) for six hours. A $125-\mu \mathrm{L}$ aliquot of plasma and $150-\mu \mathrm{L}$ aliquot of PBS were removed from the dialysis unit and matrix-matched to a final composition of 50:50 (v:v) plasma:PBS, in Rain-X® treated 96-well plates. The matrix-matched samples were immediately frozen at $-80{ }^{\circ} \mathrm{C}$. The protein binding assay and sample analysis were conducted at Charles River Laboratories, Inc. (Worcester, MA, USA). Samples were analyzed for brigatinib concentrations using liquid chromatography with tandem mass spectrometry (LC-MS/MS) methods that have been previously reported [11] using a dual-range assay with a lower limit of quantitation of $0.100 \mathrm{ng} / \mathrm{mL}$ and an upper limit of quantitation of $500 \mathrm{ng} / \mathrm{mL}$.

\section{Pharmacokinetics assay}

Plasma and urine samples were analyzed by Charles River Laboratories, Inc. (Worcester, MA, USA) by LC-MS/MS, as previously reported [11]. Plasma samples were analyzed using a dual-range assay with a lower limit of quantitation of 0.100 $\mathrm{ng} / \mathrm{mL}$ and an upper limit of $2500 \mathrm{ng} / \mathrm{mL}$ [11]. Urine samples were analyzed using a single-range assay with a lower limit of quantitation of $25.0 \mathrm{ng} / \mathrm{mL}$ and upper limit of $2500 \mathrm{ng} / \mathrm{mL}$ [11].

\section{Safety}

Adverse events were recorded throughout the study. Physical examinations were performed at screening and on days -1 and 8; height, weight, and BMI were collected only at screening. Vital signs were recorded at screening; on day -1 ; at 0.5 , $1,2,3,4,6,8,12,24,36,48,60,72,96,120$, and $144 \mathrm{~h}$ after brigatinib administration; and on day 8 . Electrocardiograms were conducted at screening, day -1 , before the brigatinib dose, 3 and $48 \mathrm{~h}$ after brigatinib, and on day 8. Clinical laboratory evaluations were performed at screening and on days 1 and 8.

\section{Pharmacokinetic data analysis}

Individual participant plasma concentration-time profiles were analyzed via noncompartmental analysis methods using Phoenix WinNonlin (version 6.4) to estimate PK parameters. Plasma PK parameters calculated for brigatinib included maximum observed plasma concentration $\left(\mathrm{C}_{\max }\right) ; \mathrm{T}_{\max } ;$ area under the plasma concentration-time curve from time zero to the time of the last measurable concentration $\left(\mathrm{AUC}_{0}\right.$ - last $)$; $\mathrm{AUC}$ from time zero to infinity $\left(\mathrm{AUC}_{0-\infty}\right)$; terminal elimination half-life $\left(\mathrm{t}_{1 / 2}\right)$; apparent oral clearance $(\mathrm{CL} / \mathrm{F})$; and apparent volume of distribution (V/F). The fraction of unbound brigatinib in plasma was determined using the following formula: $\%$ free (unbound) $=$ [free brigatinib concentration measured in buffer after RED]/[total concentration measured in plasma] X 100. Unbound brigatinib plasma PK parameters (except unbound $\mathrm{CL} / \mathrm{F}$ and $\mathrm{V} / \mathrm{F}$, which were calculated as total $\mathrm{CL} / \mathrm{F}$ or total $\mathrm{V} / \mathrm{F}$ divided by the overall mean fraction unbound) were calculated using the following formula: unbound PK parameter $=($ parameter based on total concentrations $) \mathrm{x}$ (individual participant's overall mean fraction unbound value). Urinary PK parameters included the amount of brigatinib excreted from time zero to time $\mathrm{t}\left(\mathrm{Ae}_{0-\mathrm{t}}\right)$, fraction of the dose excreted $(\%)$ from time zero to time $\mathrm{t}\left(\mathrm{Fe}_{0-\mathrm{t}}\right)$, and renal clearance $\left(\mathrm{CL}_{\mathrm{R}}\right)$. 
Table 1 Demographics and baseline characteristics

\begin{tabular}{lll}
\hline Characteristic & $\begin{array}{l}\text { Normal Renal Function } \\
(\mathrm{n}=8)\end{array}$ & $\begin{array}{l}\text { Severe Renal Impairment } \\
(\mathrm{n}=8)\end{array}$ \\
\hline Age, mean (SD), years & $59.8(9.1)$ & $61.8(7.3)$ \\
Male, $\mathrm{n}(\%)$ & $6(75.0)$ & $6(75.0)$ \\
Race, $\mathrm{n}(\%)$ & $5(62.5)$ & $7(87.5)$ \\
White & $3(37.5)$ & $1(12.5)$ \\
Black or African American & $1(12.5)$ & $3(37.5)$ \\
Ethnicity, n (\%) & $7(87.5)$ & $5(62.5)$ \\
Hispanic or Latino & $32.5(3.4)$ & $33.1(3.9)$ \\
Not Hispanic or Latino & & $7(87.5)$ \\
BMI, mean (SD), kg/m ${ }^{2}$ & $7(87.5)$ & 0 \\
Smoking habit, $\mathrm{n}(\%)$ & $1(12.5)$ & $1(12.5)$ \\
Never smoked & 0 & $3.5(1.1)$ \\
Current smoker & $0.8(0.1)$ & $18.3(4.4)$ \\
Former smoker & $107.4(10.2)$ & $10-26$ \\
Serum creatinine, mean (SD), mg/dL & $90-122$ &
\end{tabular}

BMI, body mass index; eGFR, estimated glomerular filtration rate; SD, standard deviation

\section{Statistical analysis}

Descriptive statistics, including number of observations (n), arithmetic mean, standard deviation (SD), coefficient of variation (CV\%), median, minimum, and maximum, were calculated for each time point for brigatinib plasma concentrations in each study group. Arithmetic mean; SD; $\mathrm{CV} \%$; median, minimum, and maximum values; and geometric mean and geometric CV\% were calculated for all
PK parameters except $T_{\max }$, which was reported as median (minimum, maximum). Statistical comparisons of brigatinib PK parameters (unbound) were made using a one-factor analysis of variance, and $90 \%$ CIs for the geometric mean ratios (GMRs) were calculated for the comparison of $\mathrm{C}_{\text {max }}, \mathrm{AUC}_{0-\text { last }}$, and $\mathrm{AUC}_{0-\infty}$ between study groups. Descriptive statistics were used to present safety outcomes, including number and percentage of participants with adverse events.
Fig. 1 Mean (standard deviation) fraction unbound for brigatinib at 2,8 , and $24 \mathrm{~h}$ postdose by renal function group






\section{Results}

\section{Demographics and baseline characteristics}

A total of 16 participants were screened and enrolled in the study, including eight patients with severe renal impairment and eight matched healthy volunteers with normal renal function. All participants completed the study, and no participants were excluded from any analyses. Demographic and baseline characteristics were generally similar in both study groups (Table 1). Age ranged from 47 to 70 years in both groups, with a mean of 60 years in healthy volunteers with normal renal function and 62 years in patients with severe renal impairment. Most participants were male, white, and had never smoked. Baseline eGFR ranged from 90 to $122 \mathrm{~mL} / \mathrm{min} / 1.73$ $\mathrm{m}^{2}$ for healthy volunteers with normal renal function and from 10 to $26 \mathrm{~mL} / \mathrm{min} / 1.73 \mathrm{~m}^{2}$ for patients with severe renal impairment. Results were similar when renal impairment was categorized by eGFR using the MDRD equation or creatinine clearance using the Cockcroft-Gault equation.

\section{Plasma protein binding of brigatinib}

Blood samples were collected at 2, 8 , and $24 \mathrm{~h}$ postdose to assess the plasma protein binding of brigatinib. The mean fraction unbound for brigatinib was similar across the three time points for both renal function groups, indicating that protein binding was not concentration-dependent (Fig. 1). Accordingly, the plasma protein binding data were averaged across the three time points for each participant to calculate a mean unbound value for the subsequent derivation of unbound plasma PK parameters for brigatinib. There was no effect of severe renal impairment on brigatinib plasma protein binding, as the overall mean (SD) fraction bound was $91 \%$ $(2.2 \%)$ for healthy volunteers with normal renal function and $92 \%(1.4 \%)$ for patients with severe renal impairment.
Fig. 2 Mean (standard deviation) unbound brigatinib plasma concentration-time profiles from (a) 0 to $24 \mathrm{~h}$ postdose, linear scale and (b) 0 to $168 \mathrm{~h}$ postdose, loglinear scale

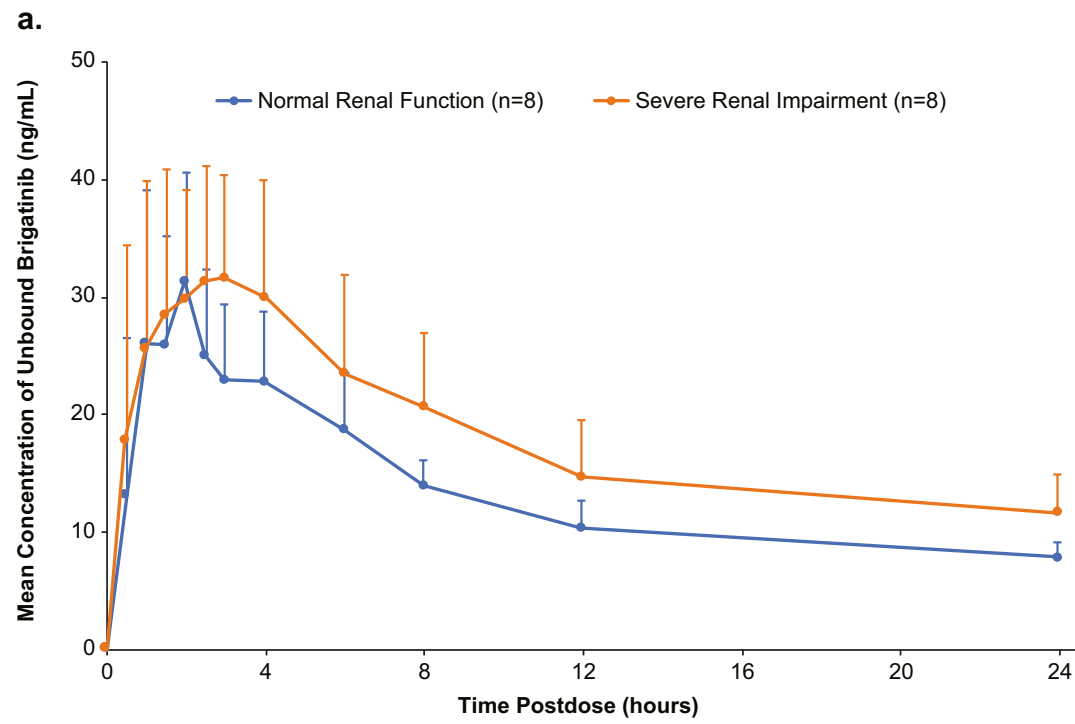

b.

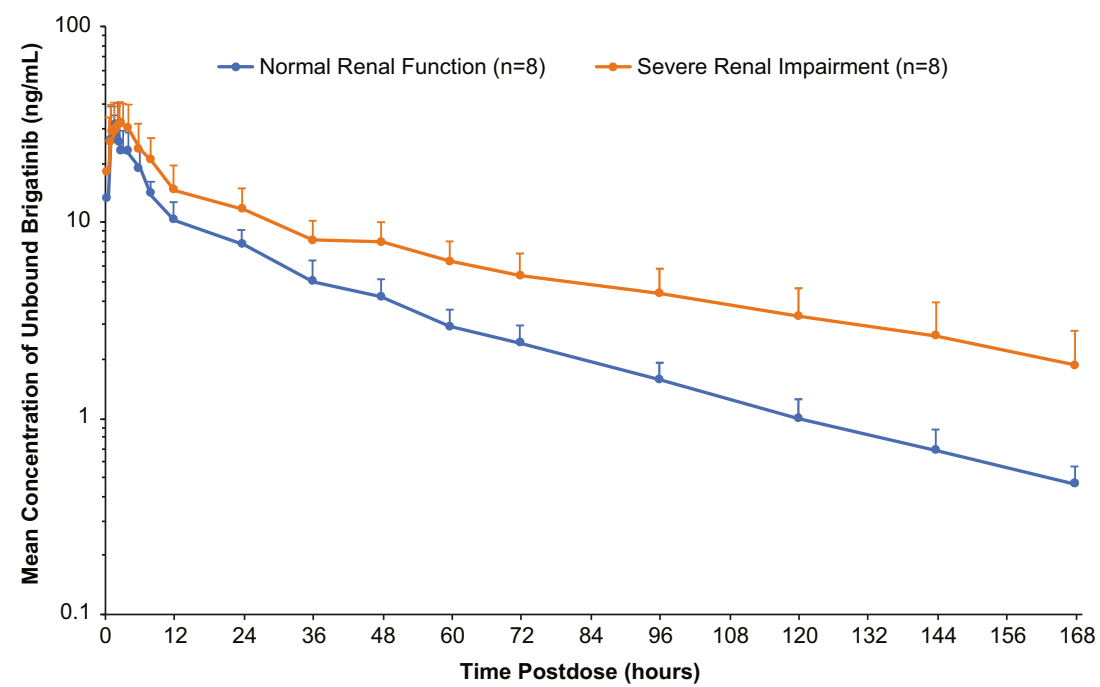


Table 2 Plasma pharmacokinetic parameters for unbound brigatinib

\begin{tabular}{|c|c|c|}
\hline Parameter $^{\mathrm{a}}$ & $\begin{array}{l}\text { Normal Renal } \\
\text { Function } \\
(n=8)\end{array}$ & $\begin{array}{l}\text { Severe Renal } \\
\text { Impairment } \\
(\mathrm{n}=8)\end{array}$ \\
\hline $\mathrm{C}_{\text {max }, \mathrm{u}}(\mathrm{ng} / \mathrm{mL})$ & $32.29(25.1)$ & $36.76(29.0)$ \\
\hline Range & $23.9-45.3$ & $25.2-55.6$ \\
\hline $\mathrm{AUC}_{0-\text { last,u }}(\mathrm{h} \cdot \mathrm{ng} / \mathrm{mL})$ & $613.1(22.4)$ & $1086(27.3)$ \\
\hline Range & $438-847$ & $726-1544$ \\
\hline $\mathrm{AUC}_{0-\infty, \mathrm{u}}(\mathrm{h} \cdot \mathrm{ng} / \mathrm{mL})$ & $641.4(22.2)$ & $1230(30.6)$ \\
\hline Range & $460-883$ & 799-2017 \\
\hline $\mathrm{CL} / \mathrm{F}, \mathrm{u}(\mathrm{L} / \mathrm{h})$ & $140.3(22.2)$ & $73.15(30.6)$ \\
\hline Range & $102-196$ & $44.6-113$ \\
\hline $\mathrm{V} / \mathrm{F}_{\mathrm{,u}}(\mathrm{L})$ & $8327(30.4)$ & $5532(31.4)$ \\
\hline Range & $5898-13,744$ & $3556-8914$ \\
\hline
\end{tabular}

$\mathrm{AUC}_{0-\infty, \mathrm{u}}$, unbound area under the plasma concentration-time curve from time zero to infinity; $\mathrm{AUC}_{0-\text { last, } \mathrm{u}}$, unbound area under the plasma concentration-time curve from time zero to the time of the last measurable concentration; $\mathrm{CL} / \mathrm{F}, \mathrm{u}$, unbound apparent oral clearance; $\mathrm{C}_{\max , \mathrm{u}}$, unbound maximum observed plasma concentration; $\mathrm{V} / \mathrm{F}, \mathrm{u}$, unbound apparent volume of distribution

${ }^{\text {a }}$ Data presented as geometric mean (geometric \% coefficient of variation)

\section{Plasma pharmacokinetic parameters for total brigatinib}

Evaluation of PK parameters for total brigatinib showed higher $\mathrm{C}_{\max }$ concentrations and AUC values for patients with severe renal impairment versus healthy volunteers with normal renal function. Geometric mean $(\mathrm{CV} \%)$ for total brigatinib $\mathrm{C}_{\max }$ was $471.1(27.2 \%) \mathrm{ng} / \mathrm{mL}$ for patients with severe renal impairment and $362.4(32.1 \%) \mathrm{ng} / \mathrm{mL}$ for healthy volunteers with normal renal function (GMR [90\% $\mathrm{CI}]: 1.30$ [1.00-1.68]). Brigatinib total $\mathrm{AUC}_{0-\infty}$ and $\mathrm{AUC}_{0-}$ last were more than $100 \%$ higher $\left(\mathrm{AUC}_{0-\infty}\right.$ GMR $[90 \% \mathrm{CI}]$ : 2.19 [1.75-2.74]; $\mathrm{AUC}_{0-\text { last }} \mathrm{GMR}$ [90\% CI]: 2.02 [1.652.48]) in patients with severe renal impairment compared with healthy volunteers with normal renal function.

Median (range) $\mathrm{T}_{\max }$ was 2.5 (1.0-3.0) and $2.0(1.0-4.0)$ hours in the severe renal impairment and normal renal function groups, respectively. The mean $t_{1 / 2}$ of brigatinib after a single dose was approximately $54 \mathrm{~h}$ in patients with severe renal impairment and $42 \mathrm{~h}$ in healthy volunteers with normal renal function.

\section{Plasma pharmacokinetics of unbound brigatinib}

Plasma PK parameters for unbound brigatinib showed similar trends to those for total brigatinib. Unbound plasma drug concentration-time curves are provided in Fig. 2a (0$24 \mathrm{~h}$ postdose $)$ and $\mathrm{b}(0-168 \mathrm{~h}$ postdose). After a single oral dose of brigatinib $90 \mathrm{mg}$, patients with severe renal impairment had higher unbound plasma brigatinib concentrations compared with healthy volunteers with normal renal function. PK parameters for unbound brigatinib are shown in Table 2. Geometric means for unbound brigatinib $\mathrm{C}_{\max }$ were generally comparable across the two groups (GMR [90 \% CI]: 1.14 [0.90-1.44]), whereas unbound brigatinib AUC values were higher in patients with severe renal impairment compared with healthy volunteers with normal renal function (Table 2). Specifically, unbound brigatinib $\mathrm{AUC}_{0-\text { last }}$ and $\mathrm{AUC}_{0-\infty}$ were $77 \%$ (GMR [90\% CI]: 1.77 [1.43-2.20]) and $92 \%$ (GMR [90\% CI]: 1.92 [1.52-2.42]) higher, respectively, in patients with severe renal impairment versus healthy volunteers with normal renal function (Table 3).

\section{Urinary pharmacokinetics of brigatinib}

The mean (SD) fraction of the administered brigatinib $90 \mathrm{mg}$ dose excreted from 0 to $168 \mathrm{~h}$ postdose was $8.4 \%$ (4.2) in patients with severe renal impairment and $18.8 \%$ (4.0) in healthy volunteers with normal renal function, thereby representing a reduction of approximately $50 \%$ (Online Resource 1). In addition, brigatinib renal clearance in patients with severe renal impairment was approximately $20 \%$ of that observed in healthy volunteers with normal renal function.

\section{Safety}

Two patients with severe renal impairment reported three treatment-emergent adverse events. One patient had both a

Table 3 Comparison of unbound brigatinib plasma pharmacokinetic parameters

\begin{tabular}{llll}
\hline Parameter & Comparison & LS Mean (SE) & Geometric LS Mean Ratio (90\% CI) \\
\hline $\mathrm{C}_{\text {max,u }}(\mathrm{ng} / \mathrm{mL})$ & Severe renal impairment vs. Normal renal function & $0.130(0.1330)$ & $1.14(0.90-1.44)$ \\
$\mathrm{AUC}_{0-\text { last,u }}(\mathrm{h} \cdot \mathrm{ng} / \mathrm{mL})$ & & $0.572(0.1229)$ & $1.77(1.43-2.20)$ \\
$\mathrm{AUC}_{0-\infty, \mathrm{u}}(\mathrm{h} \cdot \mathrm{ng} / \mathrm{mL})$ & & $0.652(0.1311)$ & $1.92(1.52-2.42)$ \\
\hline
\end{tabular}

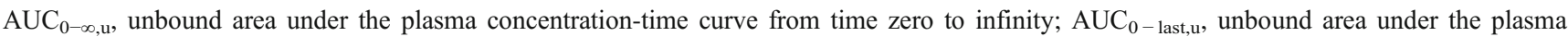
concentration-time curve from time zero to the time of the last measurable concentration; $\mathrm{CI}$, confidence interval; $\mathrm{C}_{\text {max,u }}$, unbound maximum observed plasma concentration; LS, least squares; SE, standard error 
serious adverse event hip fracture and a mild skin abrasion; both were considered unlikely to be related to study drug by the investigator. The second patient experienced an influenzalike illness, which was assessed as mild and possibly related to study drug by the investigator. Both patients completed the study. One additional patient with severe renal impairment had a clinically significant elevated systolic blood pressure postdose on day 1 , but also had a medical history of hypertension. No healthy volunteers with normal renal function reported adverse events. There were no treatment-emergent adverse events related to clinical laboratory results reported, and no participant had clinically significant laboratory values or electrocardiogram findings during the study.

\section{Discussion}

The clearance of the ALK inhibitor brigatinib is characterized by a major contribution of cytochrome $\mathrm{P} 450$-mediated metabolism and a minor contribution of renal clearance. Accordingly, the purpose of this study was to evaluate the impact of severe renal impairment on brigatinib PK to complement population PK model-based assessments of brigatinib PK in mild or moderate renal impairment. Although unbound maximum plasma concentrations of brigatinib were comparable between renal function groups following single-dose oral administration, total systemic exposure of unbound brigatinib $\left(\mathrm{AUC}_{0-\infty}\right)$ was approximately $92 \%$ higher in patients with severe renal impairment compared with healthy volunteers with normal renal function. Severe renal impairment had no effect on brigatinib plasma protein binding. The renal clearance of unbound brigatinib was approximately five-fold lower in patients with severe renal impairment compared with that in healthy volunteers with normal renal function. The observation of an approximately $92 \%$ higher total systemic exposure of unbound brigatinib in severe renal impairment supports a brigatinib dosage reduction of approximately $50 \%$ (i.e., from $180 \mathrm{mg}$ to $90 \mathrm{mg}$ or from $90 \mathrm{mg}$ to $60 \mathrm{mg}$ ) for patients with severe renal impairment to result in unbound systemic exposures comparable to those achieved in patients with normal renal function.

The data from this study add to the information on the PK and dosing of brigatinib across clinical contexts of use. Brigatinib displays dose-linear and time-independent PK, with biphasic elimination and a mean plasma half-life of ap-

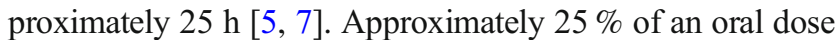
of brigatinib is excreted unchanged in the urine [12]. Mild or moderate renal impairment was not associated with clinically meaningful effects on brigatinib PK based on the results of a population PK analysis; consequently, no dosage adjustments are required in patients with eGFR $\geq 30 \mathrm{~mL} / \mathrm{min} / 1.73 \mathrm{~m}^{2}$ [9]. In addition, a high-fat meal was shown to have no effect on the total exposure (AUC) of brigatinib, and thus the drug can be administered without regard to meals [13]. Brigatinib is primarily metabolized by CYP3A4, with minor contribution by $2 \mathrm{C} 8$ in vitro [11]. Systemic exposures (AUC) of brigatinib were found to increase by $101 \%$ with coadministration of strong CYP3A inhibitors and decrease by $80 \%$ with strong CYP3A inducers; therefore, concomitant use of these agents with brigatinib is not recommended [11]. When concomitant use of brigatinib with a strong CYP3A inhibitor cannot be avoided, the brigatinib dose should be reduced by approximately $50 \%$ [11]. In contrast, coadministration of brigatinib with a strong inhibitor of CYP2C8 did not meaningfully affect brigatinib systemic exposures (AUC), and no dose modifications are therefore needed when brigatinib is coadministered with strong CYP2C8 inhibitors [11].

The results of this study indicate that both renal and nonrenal clearance of brigatinib are impacted in severe renal impairment. Renal clearance of brigatinib in patients with severe renal impairment was $20 \%$ of that in healthy volunteers with normal renal function. As $\mathrm{C}_{\max }$ was not meaningfully different between the renal function groups, it appears that bioavailability is not affected by renal impairment. Considering that renal clearance accounts for approximately $25 \%$ of overall clearance of brigatinib, it can thus be derived that nonrenal clearance of brigatinib in severe renal impairment is approximately $50 \%$ lower than in the setting of normal renal function. Although the precise mechanism of the effects of renal impairment on the nonrenal clearance of brigatinib is unknown, the results of this study provide the scientific basis for appropriate dosage of brigatinib in order to achieve a favorable benefit versus risk profile during treatment of patients with ALK + NSCLC who have severe renal insufficiency.

In conclusion, this study showed that brigatinib exposure is higher in patients with severe renal impairment compared with healthy volunteers with normal renal function and supports an approximate $50 \%$ dose reduction in this patient population, as reflected in the prescribing information $[5,14]$.

Supplementary Information The online version contains supplementary material available at https://doi.org/10.1007/s10637-021-01095-5.

Acknowledgements The authors would like to thank the study participants, their families, and their caregivers; the study investigators and their team members at the study site; and colleagues from Millennium Pharmaceuticals, Inc., Cambridge, MA, USA, a wholly owned subsidiary of Takeda Pharmaceutical Company Limited. Professional medical writing assistance was provided by Lauren Gallagher, $\mathrm{RPh}, \mathrm{PhD}$, and Lela Creutz, PhD, of Peloton Advantage, LLC, an OPEN Health company, Parsippany, NJ, USA, and funded by Millennium Pharmaceuticals, Inc.

Author contributions Study design: All authors. Principal investigator: Thomas Marbury.

Study investigator: Thomas Marbury.

Enrolled patients: Thomas Marbury.

Collection and assembly of data: Neeraj Gupta, Thomas Marbury, Michael J. Hanley. 
Data analysis and interpretation: Neeraj Gupta, Michael J. Hanley, Karthik Venkatakrishnan.

Manuscript preparation: Neeraj Gupta.

Manuscript review and revisions: All authors.

Final approval of manuscript: All authors.

Funding This study was sponsored by ARIAD Pharmaceuticals, Inc., Cambridge, MA, a wholly owned subsidiary of Takeda Pharmaceutical Company Limited.

Data availability The data sets, including the redacted study protocol, redacted statistical analysis plan, and individual participant data supporting the results reported in this article, will be made available within three months from initial request, to researchers who provide a methodologically sound proposal. The data will be provided after deidentification, in compliance with applicable privacy laws, data protection, and requirements for consent and anonymization.

Code availability Not applicable.

\section{Declarations}

Conflicts of interest/Competing interests NG: Employee of Millennium Pharmaceuticals, Inc., Cambridge, MA, a wholly owned subsidiary of Takeda Pharmaceutical Company Limited.

MJH: Employee of Millennium Pharmaceuticals, Inc., Cambridge, MA, a wholly owned subsidiary of Takeda Pharmaceutical Company Limited.

DK: Former employee and stock owner of Millennium Pharmaceuticals, Inc., a wholly owned subsidiary of Takeda Pharmaceutical Company Limited.

MT: Former employee of ARIAD Pharmaceuticals, Inc., a wholly owned subsidiary of Takeda Pharmaceutical Company Limited.

NN: Former employee of ARIAD Pharmaceuticals, Inc., a wholly owned subsidiary of Takeda Pharmaceutical Company Limited.

TCM: Employee and equity owner of Orlando Clinical Research Center

KV: Former employee of Millennium Pharmaceuticals, Inc., a wholly owned subsidiary of Takeda Pharmaceutical Company Limited.

Ethics approval Approval for this study was obtained from the institutional review board of the study site (Orlando Clinical Research Center, Orlando, FL, USA). This study was conducted in accordance with current Good Clinical Practice, the Declaration of Helsinki, the International Council for Harmonisation guidelines, and all applicable regulatory requirements.

Consent to participate All participants provided written informed consent.

\section{Consent for publication Not applicable.}

Open Access This article is licensed under a Creative Commons Attribution 4.0 International License, which permits use, sharing, adaptation, distribution and reproduction in any medium or format, as long as you give appropriate credit to the original author(s) and the source, provide a link to the Creative Commons licence, and indicate if changes were made. The images or other third party material in this article are included in the article's Creative Commons licence, unless indicated otherwise in a credit line to the material. If material is not included in the article's Creative Commons licence and your intended use is not permitted by statutory regulation or exceeds the permitted use, you will need to obtain permission directly from the copyright holder. To view a copy of this licence, visit http://creativecommons.org/licenses/by/4.0/.

\section{References}

1. Katayama R, Khan TM, Benes C, Lifshits E, Ebi H, Rivera VM, Shakespeare WC, Iafrate AJ, Engelman JA, Shaw AT (2011) Therapeutic strategies to overcome crizotinib resistance in nonsmall cell lung cancers harboring the fusion oncogene EML4ALK. Proc Natl Acad Sci USA 108(18):7535-7540. https://doi. org/10.1073/pnas.1019559108

2. Huang WS, Liu S, Zou D, Thomas M, Wang Y, Zhou T, Romero J, Kohlmann A, Li F, Qi J, Cai L, Dwight TA, Xu Y, Xu R, Dodd R, Toms A, Parillon L, Lu X, Anjum R, Zhang S, Wang F, Keats J, Wardwell SD, Ning Y, Xu Q, Moran LE, Mohemmad QK, Jang HG, Clackson T, Narasimhan NI, Rivera VM, Zhu X, Dalgarno D, Shakespeare WC (2016) Discovery of brigatinib (AP26113), a phosphine oxide-containing, potent, orally active inhibitor of anaplastic lymphoma kinase. J Med Chem 59(10):4948-4964. https:// doi.org/10.1021/acs.jmedchem.6b00306

3. Zhang S, Anjum R, Squillace R, Nadworny S, Zhou T, Keats J, Ning Y, Wardwell SD, Miller D, Song Y, Eichinger L, Moran L, Huang WS, Liu S, Zou D, Wang Y, Mohemmad Q, Jang HG, Ye E, Narasimhan N, Wang F, Miret J, Zhu X, Clackson T, Dalgarno D, Shakespeare WC, Rivera VM (2016) The potent ALK inhibitor brigatinib (AP26113) overcomes mechanisms of resistance to first- and second-generation ALK inhibitors in preclinical models. Clin Cancer Res 22(22):5527-5538

4. Huber RM, Hansen KH, Paz Ares Rodríguez L, West HL, Reckamp KL, Leighl NB, Tiseo M, Smit EF, Kim D, Gettinger SN, Hochmair MJ, Kim S, Langer CJ, Ahn M, Kim ES, Kerstein D, Groen HJM, Camidge DR (2020) Brigatinib in crizotinibrefractory ALK + NSCLC: 2-year follow-up on systemic and intracranial outcomes in the phase 2 ALTA trial. J Thorac Oncol 15(3):404-415. https://doi.org/10.1016/j.jtho.2019.11.004

5. Alunbrig brigatinib [summary of product characteristics] (2020) Takeda Pharmaceutical Company Limited. https://www.alunbrig. com/assets/pi.pdf. Accessed 9 Mar 2021

6. Camidge R, Kim HR, Ahn M, Yang JCH, Han J, Hochmair MJ, Lee KH, Delmonte A, Garcia Campelo MR, Kim D, Griesinger F, Felip E, Califano R, Spira A, Gettinger SN, Tiseo M, Lin HM, Gupta N, Hanley MJ, Ni Q, Zhang P, Popat S (2020) Brigatinib versus crizotinib in advanced ALK inhibitor-naive ALK-positive non-small cell lung cancer: Second interim analysis of the phase III ALTA-1L trial. J Clin Oncol 38(31):3592-3603. https://doi.org/10. 1200/JCO.20.00505

7. Gettinger SN, Bazhenova LA, Langer CJ, Salgia R, Gold KA, Rosell R, Shaw AT, Weiss GJ, Tugnait M, Narasimhan NI, Dorer DJ, Kerstein D, Rivera VM, Clackson T, Haluska FG, Camidge DR (2016) Activity and safety of brigatinib in $A L K$-rearranged nonsmall-cell lung cancer and other malignancies: A single-arm, open-label, phase 1/2 trial. Lancet Oncol 17(12):1683-1696

8. Gupta N, Wang X, Offman E, Rich B, Kerstein D, Hanley M, Diderichsen PM, Zhang P, Venkatakrishnan K (2020) Brigatinib dose rationale in anaplastic lymphoma kinase-positive non-small cell lung cancer: Exposure-response analyses of pivotal ALTA study. CPT Pharmacometrics Syst Pharmacol 9(12):718-730. https://doi.org/10.1002/psp4.12569

9. Gupta N, Wang X, Offman E, Prohn M, Narasimhan N, Kerstein D, Hanley MJ, Venkatakrishnan K (2021) Population pharmacokinetics of brigatinib in healthy volunteers and patients with cancer. Clin 
Pharmacokinet 60(2):235-247. https://doi.org/10.1007/s40262020-00929-4

10. Levey AS, Coresh J, Greene T, Stevens LA, Zhang YL, Hendriksen S, Kusek JW, Van Lente F (2006) Using standardized serum creatinine values in the modification of diet in renal disease study equation for estimating glomerular filtration rate. Ann Intern Med 145(4):247-254. https://doi.org/10.7326/0003-4819-145-4200608150-00004

11. Tugnait M, Gupta N, Hanley MJ, Sonnichsen D, Kerstein D, Dorer DJ, Venkatakrishnan K, Narasimhan N (2020) Effects of strong CYP2C8 or CYP3A inhibition and CYP3A induction on the pharmacokinetics of brigatinib, an oral anaplastic lymphoma kinase inhibitor, in healthy volunteers. Clin Pharmacol Drug Dev 9(2): 214-223. https://doi.org/10.1002/cpdd.723

12. Hirota T, Muraki S, Ieiri I (2019) Clinical pharmacokinetics of anaplastic lymphoma kinase inhibitors in non-small-cell lung cancer. Clin Pharmacokinet 58(4):403-420. https://doi.org/10. 1007/s40262-018-0689-7

13. Tugnait M, Gupta N, Hanley MJ, Venkatakrishnan K, Sonnichsen D, Kerstein D, Dorer DJ, Narasimhan N (2019) The effect of a high-fat meal on the pharmacokinetics of brigatinib, an oral anaplastic lymphoma kinase inhibitor, in healthy volunteers. Clin Pharmacol Drug Dev 8(6):734-741. https://doi.org/10.1002/cpdd. 641

14. (2020) Alunbrig [summary of product characteristics]. Takeda Pharma A/S, Taastrup, Denmark

Publisher's note Springer Nature remains neutral with regard to jurisdictional claims in published maps and institutional affiliations. 